\title{
Meta-Análise para Estimativas de Herdabilidade para Características de Crescimento em Bovinos de Corte ${ }^{1}$
}

\author{
Juliana Di Giorgio Giannotti ${ }^{2}$, Irineu Umberto Packer ${ }^{3}$, Maria Eugênia Zerlotti Mercadante ${ }^{4}$
}

\begin{abstract}
RESUMO - Um conjunto de estimativas de herdabilidade de características de crescimento em bovinos de corte de raças indianas, procedentes de 186 artigos publicados, foi compilado e sumarizado, utilizando-se técnicas de meta-análise. Com o objetivo de identificar quais fatores afetam as estimativas de herdabilidade ponderadas, empregou-se o método dos mínimos quadrados para análise de um modelo de efeito fixo. Os fatores estatisticamente significativos, para as quatro características, foram raça e método de estimação, sendo que este último deve ser sempre considerado na síntese dessas estimativas. Os valores das estimativas combinadas das herdabilidades, para as características peso ao nascimento, peso à desmama, peso aos 365 dias e peso aos 550 dias obtidos foram: $0,30 \pm 0,01 ; 0,23 \pm 0,01$; $0,27 \pm 0,01$; e $0,31 \pm 0,01$, respectivamente. A meta-análise é uma técnica recomendada para resumir informações sobre parâmetros genéticos de populações bovinas.
\end{abstract}

Palavras-chave: estimativas combinadas, parâmetros genéticos, zebuínos

\section{Meta-Analysis for Heritability of Estimates Growth Traits in Beef Cattle}

\begin{abstract}
A meta-analysis was conducted to summarize published heritability of estimates growth traits of zebu beef cattle of 186 reports. The factors affecting weighed heritability estimates were analyzed by the least square means method for a model including fixed effects. Breed and estimation method were the significant factors in the four traits (birth weight, weaning weight, weight at 365 days and weight at 550 days). Estimation method is always recommeded to summarize these estimates. The pooled heritability estimates for birth weight, weaning weight, weight at 365 days old and weight at 550 days old were $0.30 \pm 0.01,0.23 \pm 0.01,0.27 \pm 0.01$ and $0.31 \pm 0.01$, respectively. Meta-analysis techniques were recommended for polling of genetic parameters of zebu beef cattle.
\end{abstract}

Key Words: genetic parameters, pooled estimates, zebu cattle

\section{Introdução}

A meta-análise pode ser definida como um procedimento estatístico que consiste de uma revisão quantitativa e resumida de resultados de estudos distintos, mas relacionados (Glass, 1976). Os métodos estatísticos empregados na meta-análise asseguram a obtenção de uma estimativa combinada e precisa, sobretudo em virtude do aumento do número de observações e, conseqüentemente, do poder estatístico e da possibilidade de examinar a variabilidade entre os estudos (Fagard et al., 1996).

$\mathrm{Na}$ pesquisa científica, é crescente o número de artigos similares conduzidos e publicados nas diversas áreas do conhecimento, gerando interesse, muitas vezes, de realizar síntese destes resultados (Fagard et al., 1996). Desse modo, a aplicação da meta-análise tem aumentado em vários campos das ciências (Cooper,
1989): agronomia (Olkin \& Shaw, 1995), ciências sociais (Glass, 1976), ecologia (Gurevitch \& Hedges, 1993), engenharia (Pickard et al., 1998), medicina (Canner, 1987; Normand, 1999), melhoramento animal (Giannotti et al., 2002) e zootecnia (Beckett \& Lean, 1997).

Existe, no melhoramento animal, como nas demais áreas, o anseio de sumarizar o conhecimento adquirido no formato de revisão. Esta pretensão ocorre, principalmente, em virtude do aumento do conhecimento científico nesta área e da necessidade do pesquisador em entender as relações biológicas, de ambiente e de comportamento animal (St-Pierre, 2001).

Ainda no contexto do melhoramento animal, são freqüentes as revisões bibliográficas cujo objetivo é obter uma estimativa combinada, proveniente de resultados de estudos individuais. Deste modo, estimativas de parâmetros genéticos de populações bovinas, 
como correlações genéticas e fenotípicas, herdabilidade e heterose, são compiladas e resumidas, para diversas características de interesse econômico na produção animal. Nestas revisões, são empregados diferentes critérios para se obter uma estimativa combinada, destacando-se a média ponderada pelo inverso da variância (Koots et al., 1994ab; Lôbo et al., 2000) ou do desvio-padrão (Mohiuddin et al., 1993; Mercadante et al., 1995) individual de cada estimativa de parâmetro genético e a análise de regressão linear múltipla, com aplicação de análise de agrupamento (Roughsedge et al., 2001).

Porém, apesar de algumas dessas revisões constarem de pesquisa bibliográfica abrangente, obterem uma medida resumida comum para diferentes estudos e ponderarem pelo inverso da variância, em nenhuma delas foi considerada a variância existente entre os estudos. Os procedimentos estatísticos empregados em meta-análise propõem considerar o efeito da variação entre esses estudos, para a obtenção da estimativa combinada (DerSimonian \& Laird, 1986; Normand, 1999; Engels et al., 2000). A variação entre os estudos é proveniente, por exemplo, de diferentes tipos de manejo alimentar (a pasto, confinamento, semiconfinamento), raças, sexo, métodos distintos de estimação dos parâmetros envolvidos e dados oriundos de vários países (Koots et al., 1994a).

A herdabilidade, um parâmetro populacional essencial na avaliação genética animal e no delineamento de programas de melhoramento animal, é definida como uma medida do grau de semelhança dos filhos, em relação ao desempenho de seus pais, para determinada característica (Bourdon, 2000). Fatores como raça, origem dos dados (país), método de estimação e data de publicação, entre outros, podem interferir em sua obtenção $\mathrm{O}$ número de trabalhos publicados contendo estimativas de herdabilidade $\left(\hat{h}^{2}\right)$ para características de crescimento em bovinos de corte, é suficientemente grande para se conduzir análises visando determinar que fatores afetam essas características e fazer recomendações considerando valores combinados apropriados (Koots et al., 1994a).

Este estudo foi conduzido com os seguintes objetivos: i) desenvolver e aplicar a metodologia da metaanálise ao problema de resumir informações das estimativas de herdabilidade $\left(\hat{h}^{2}\right)$ para as características peso ao nascimento (PN), peso à desmama (PD), peso aos 365 dias (P365) e peso aos 550 dias
(P550) em bovinos de corte de origem indiana; ii) estimar a variância entre os estudos e incorporá-la na obtenção das estimativas combinadas das herdabilidades $\left(\hat{h}_{i+}^{2}\right)$ para as quatro características; iii) identificar os fatores que afetam as $\hat{h}^{2}$ ponderadas.

\section{Material e Métodos}

Os dados utilizados neste trabalho referem-se a $869 \hat{h}^{2}$ das características de crescimento PN, PD, P365 e P550, obtidas em populações de bovinos de corte de origem indiana (182 de PN, 331 de PD, 153 de P365 e 203 de P550), procedentes de 186 artigos publicados, dos quais 102 constam no trabalho de revisão desenvolvido por Mercadante et al. (1995) e 84 são provenientes de pesquisa bibliográfica realizada de 1995 a 2003 para a atualização dos dados. A pesquisa supracitada englobou anais de congressos, teses e dissertações e base de dados, como CAB e AGRIS, utilizando-se as palavras-chave "nelore", "nellore", "zebu", "bos indicus" e "heritabili*".

A meta-análise das $\hat{h}^{2}$ envolveu os seguintes passos: análise exploratória do conjunto de dados; verificação das pressuposições estatísticas requeridas; realização do teste de homogeneidade; estimação da variância entre os estudos; obtenção da estimativa combinada.

A análise do conjunto de dados visa detectar a presença de vício de publicação, isto é, se a pesquisa bibliográfica não foi bastante abrangente, e a presença de observações discrepantes. O vício de publicação foi examinado por meio do gráfico de funil, que consiste de um diagrama de dispersão das $\hat{h}^{2}$ versus o tamanho da amostra $(N)$. Este exame é baseado no fato da $i$-ésima estimativa de herdabilidade $\left(\hat{h}^{2}, i=1,2, \ldots, k\right.$, em que $k$ é o número de disponível para cada característica) ser tanto mais precisa quanto maior for o $N$, e de estudos com menores $N$ serem prevalecentes em qualquer pesquisa de literatura. $\mathrm{Na}$ ausência de vício de publicação, a forma do gráfico será semelhante a um funil invertido e simétrico (Egger \& Smith, 1998). As observações discrepantes foram detectadas por intermédio do dispositivo gráfico "box-plot".

A validade dos procedimentos adotados na metaanálise requer que as pressuposições de independência e normalidade das estimativas a serem combinadas sejam satisfeitas (Hedges \& Olkin, 1985). A independência é, em parte, garantida pelo fato de as 
estimativas serem obtidas de diferentes trabalhos publicados, enquanto a normalidade deve ser testada. O teste de Shapiro-Wilk, cuja hipótese de nulidade expressa que os dados em questão têm distribuição normal, foi utilizado para verificar a pressuposição de normalidade (Shapiro \& Wilk, 1965). Na ausência de normalidade, os dados foram transformados por meio do SAS (SAS, 2002), empregando-se o algoritmo sugerido por Box \& Cox (1964), cujo parâmetro de transformação $(\lambda)$ é selecionado pelo método da máxima verossimilhança.

A informação da variância $\left(s_{i}^{2}\right)$ associada a $\hat{h}^{2}$ é essencial em um estudo deste tipo, pois será usada na obtenção da estimativa combinada. Entretanto, alguns estudos não fornecem a $s_{i}^{2}$ da $\hat{h}^{2} \mathrm{e}$, para o cálculo destas, foi utilizado o método descrito por Koots et al. (1994a) e Falconer \& Mackay (1996).

Há duas formas de se combinar as informações, as quais diferem quanto à modelagem da variação entre as estimativas, de modo que a decisão acerca de qual modelo adotar é tomada com base no teste de homogeneidade. Assim, de posse das $\hat{h}^{2}$ e $s_{i}^{2}$, foi testada a hipótese $H_{0}: h_{1}^{2}=h_{2}^{2}=\ldots=h_{k}^{2}$ contra a hipótese de que pelo menos uma estimativa de herdabilidade diferia das demais. Este teste se baseia na estatística $Q \sim \chi_{k-1}^{2}$, dada por $Q=\sum_{i=1}^{k} w_{i}\left(\hat{h}_{i}^{2}-\hat{h}_{+}^{2}\right)^{2}$, em que $w_{i}=1 / s_{i}^{2}$ e $\hat{h}_{+}^{2}=\sum_{i=1}^{k} w_{i} \hat{h}_{i}^{2} / \sum_{i=1}^{k} w_{i}$ (Hedges $\&$ Olkin, 1985), realizado pelo procedimento GLM(SAS, 1990). A rejeição de $H_{0}$ sugere a não-existência da homogeneidade entre as $\hat{h}^{2}$, adotando-se, assim, um modelo de efeito aleatório, em que a variância existente entre os estudos $\left(\tau^{2}\right)$, deve ser estimada e incluída na obtenção da estimativa combinada das herdabilidades $\left(\hat{h}_{+}^{2}\right)$ (Hedges \& Olkin, 1985; Wang \& Bushman, 1999).

O modelo de efeito aleatório adotado teve como estrutura $h_{i}^{2}=h_{+}^{2}+\varepsilon_{i}+e_{i}$, em que: $\hat{h}_{i}^{2}$ representa o valor da $i$-ésima herdabilidade de determinada característica $\left(h_{i}^{2} \stackrel{\text { indep. }}{\sim} \operatorname{Normal}\left(h_{+}^{2}, s_{i}^{2}+\tau^{2}\right)\right) ; h_{+}^{2}$ representa o valor da estimativa combinada das herdabilidades; $e_{i}$ é o erro aleatório $\left(e_{i} \stackrel{\text { indep. }}{\sim} \operatorname{Normal}\left(0, s_{i}^{2}\right)\right)$; e $\varepsilon_{i}$, é o erro aleatório $\left(\varepsilon_{i} \stackrel{\text { indep. }}{\sim} \operatorname{Normal}\left(0, \tau^{2}\right)\right)($ Brockwell \& Gordon, 2001).

A variância entre estudos foi estimada pelo método da Máxima Verossimilhança Restrita $(M V R)$ em que o estimador $\hat{\tau}^{2}$ é solução para $\hat{\tau}^{2}=\sum_{i=1}^{k} w_{i}^{2^{*}}\left[\frac{k}{k-1}\left(\hat{h}_{i}^{2}-\hat{h}_{i}^{2^{*}}\right)^{2}-s_{i}^{2}\right] / \sum_{i=1}^{k} w_{i}^{2^{*}}$, pelo procedimento MIXED (SAS, 1997). Portanto, a estimativa combinada das herdabilidades, quando existe heterogeneidade entre os estudos, será encontrada a partir da equação: $\hat{h}_{+}^{2^{*}}=\sum_{i=1}^{k} w_{i}^{*} h_{i}^{2} / \sum_{i=1}^{k} w_{i}^{*}$, em que a ponderação é $w_{i}^{*}=1 /\left(s_{i}^{2}+\hat{\tau}^{2}\right)$.

O desvio-padrão combinado, associado a $\hat{h}_{+}^{2}$, foi estimado pela equação $s_{+}=\sqrt{1 / \sum_{i=1}^{k}\left(1 / w_{i}^{*}\right)}$, quando se trabalhou com um modelo de efeito aleatório, ou utilizando $w_{i}$ em vez de $w_{i}{ }^{*}$, no modelo de efeito fixo (Koots et al., 1994a).

Realizados os procedimentos de meta-análise, isto é, obtidos os valores de $\hat{\tau}^{2}$ e $\hat{h}_{+}^{2}$ para as quatro características, prosseguiu-se à análise para identificar quais fatores (raça, método de estimação e país) afetaram significativamente as ponderadas por $w_{i}$ ou $w_{i}^{*}$. Para tanto, realizou-se uma análise pelo método de mínimos quadrados, considerando-se o seguinte modelo:

$$
\hat{h}_{i j k l}^{2}=\mathrm{m}+\mathrm{R}_{\mathrm{i}}+\mathrm{M}_{\mathrm{j}}+\mathrm{P}_{\mathrm{k}}+\mathrm{e}_{\mathrm{ijk} \mathrm{l}}
$$

em que: $\hat{h}_{i j k l}^{2}$ é a estimativa de herdabilidade ponderada para determinada característica; $R_{i}$ é a raça, $i=$ $1,2, \ldots, 15$ para $\mathrm{PN} ; i=1,2, \ldots, 14$ para $\mathrm{PD} ; i=1$, $2, \ldots, 10$ para P365 e P550 (Afrikaner, Bali, Boran, Brahman, Bunaji, Gir, Gobra, Gudali, Guzerá, Indu, Kedah, Mashona, Nelore, Sahiwal, Tabapuã, Wakna e Zebu); $M_{j}$ é o método de estimação, $j=1$, 2, 3 para PN, PD e P550; $j=1,2$ para P365 (modelo animal, modelo touro e regressão pai progênie); $P_{k}$ é o país, $k=1,2, \ldots, 16$ para $\mathrm{PN}$ e $\mathrm{PD} ; k=1,2, \ldots, 11$ para P365; e $k=1,2, \ldots, 12$ para P550 (África do Sul, Austrália, Brasil, Camarão, Colômbia, Costa Rica, Cuba, Estados Unidos, Etiópia, Guatemala, Guiana, Malásia, México, Nigéria, Paquistão, Senegal, Tanzânia, Venezuela e Zimbabue). 


\section{Resultados e Discussão}

Nas quatro características, as $\hat{h}^{2}$ (Figura 1) apresentam distribuição assimétrica à direita, possuem a maior parte de seus valores com $\hat{h}^{2}$ de até 0,35 , existem poucos valores abaixo de 0,10 e acima de 0,60 . Os valores mais baixos das $\hat{h}^{2}$ foram encontrados na característica PD e os mais elevados, na característica P550. Foram detectadas como discrepantes as $\hat{h}^{2}$ cujos valores foram acima de 0,69 para PN e P365, 0,60 para PD e 0,79 para P550. Apesar destes valores serem discrepantes nas amostras das quatro características, nenhum critério de exclusão foi imposto sobre eles, pois, nos trabalhos originais, os autores fizeram considerações das razões pelas quais obtiveram valores elevados de herdabilidade para características de crescimento. Esse procedimento corrobora o adotado por Lôbo et al. (2000).

O gráfico de funil (Figura 2) comprova que não houve vício de publicação na pesquisa bibliográfica realizada, uma vez que, para as quatro características em estudo, o gráfico apresenta uma forma aproximada de funil invertido, expressando a abrangência da revisão de literatura para a inclusão dos estudos contendo as $\hat{h}^{2}$ na análise. Observou-se ainda que, nas quatro características, os tamanhos amostrais $(N)$ referentes às $\hat{h}^{2}$ apresentam maior concentração de valores entre 300 e 15.000 , com poucos abaixo de 100 e acima de 50.000 .

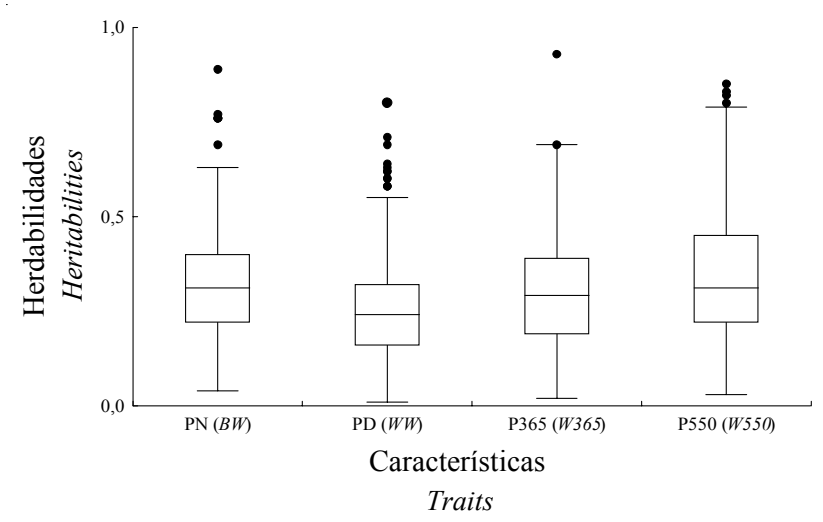

Figura 1 - Box-plot das estimativas de herdabilidade para as características peso ao nascimento $(\mathrm{PN})$, peso à desmama (PD), peso aos 365 dias (P365) e peso aos 550 dias (P550).

Figure 1 - Box-plot of the heritability estimates for birth weight $(B W)$, weaning weight (WW), weight at 365 days (W365) and weight at 550 days (W550).
Os valores da estatística do teste de Shapiro-Wilk para normalidade foram: 0,96 para $\mathrm{PN}(\mathrm{p} \leq 0,01) ; 0,94$ para PD $(\mathrm{p} \leq 0,01) ; 0,95$ para P365 e P550 ( $\leq \leq 0,01)$. Há, portanto, forte evidência de que os dados em questão não seguem distribuição normal, havendo a necessidade de transformação desses dados para aproximar a normalidade e garantir o poder dos testes estatísticos.

A transformação das $\hat{h}^{2}$ se deu elevando cada uma das $\hat{h}^{2}$ à potência de $l=0,40$, pois este valor promoveu a minimização da soma de quadrados do resíduo do modelo. O teste de Shapiro-Wilk para normalidade foi realizado para as $\hat{h}^{2}$ transformadas e o valor da estatística do teste foi $0,99(p>0,05)$ para as quatro características, evidenciando a decisão de não-rejeição da hipótese de normalidade, considerando-se um nível mínimo de 0,05 de significância.

O prosseguimento da análise se deu com o teste de homogeneidade, utilizando-se as $\hat{h}^{2}$ transformadas (Tabela 1). A hipótese de homogeneidade entre as $\hat{h}^{2}$ transformadas foi rejeitada para as quatro características $(p \leq 0,01)$ e, nestas condições, um modelo de efeito aleatório foi mais adequado.

Portanto, adotando-se um modelo de efeito aleatório, foi necessário estimar a variância entre os estudos ( $\left.\hat{\tau}^{2}\right)$, resultando em: 0,0069 para PN; 0,0059 para PD; 0,0070 para P365; e 0,0084 para P550. As $\hat{h}^{2}$ foram combinadas utilizando-se a ponderação $w_{i}^{*}$. Os valores das $\hat{h}_{+}^{2}$ e seus respectivos $\hat{s}_{+}$, para as características PN, PD, P365 e P550 encontram-se na Tabela 2.

A magnitude das $\hat{h}^{2}$ é importante, pois, se uma característica é altamente herdável, pode-se afirmar que animais com alto ou baixo desempenho tenderão a produzir filhos com alto ou baixo desempenho. Por outro lado, se uma característica não é muito herdável, registros de desempenho dos pais revelarão pouco sobre o desempenho da progênie.

Quando se comparam as $\hat{h}_{+}^{2}$ obtidas neste trabalho com outros trabalhos, verifica-se que estes valores estão bastante próximos. Bourdon (2000) considera como referência os valores 0,40 para $\mathrm{PN}$ e P365, 0,30 para PD e 0,65 para P550. Koots et al. (1994a), utilizando a ponderação $w_{i}$ para combinar $\hat{h}^{2}$ para características de crescimento em bovinos de corte de raças zebuínas, européias e cruzamentos, obtiveram $\hat{h}_{+}^{2}$ de $0,31 \pm 0,003$, para PN, 0,24 $\pm 0,002$, para $\mathrm{PD}$, e de $0,33 \pm 0,004$, para $\mathrm{P} 365$. Lôbo et al. (2000) também utilizando a ponderação $w_{i}$, para combinar $\hat{h}^{2}$ para características de crescimento em bovinos de corte de raças zebuínas, européias e 

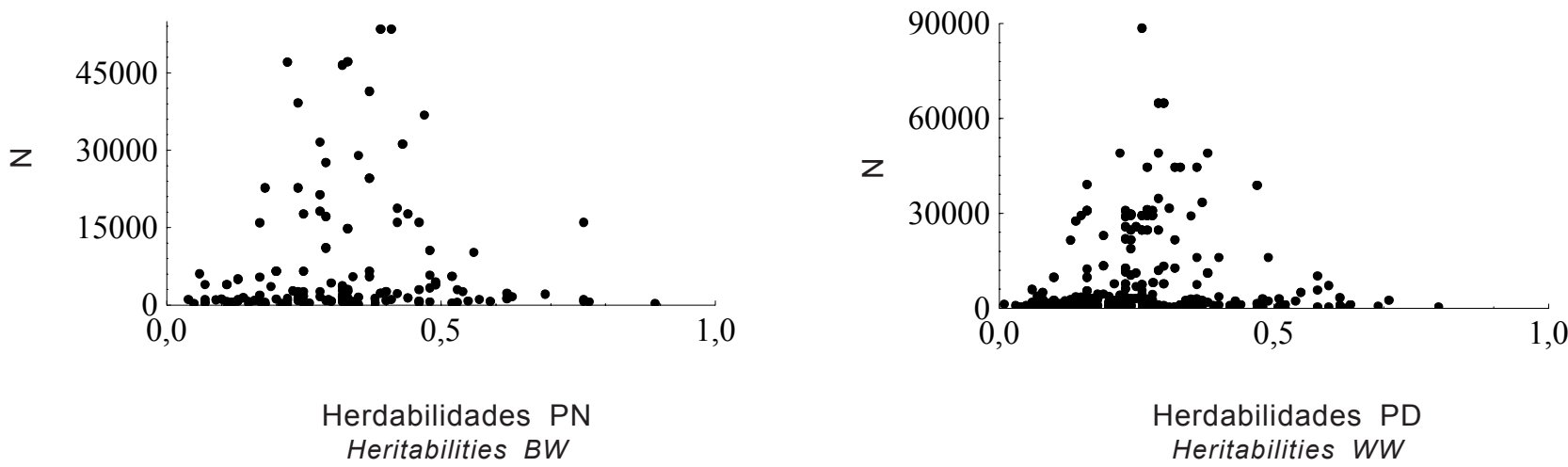

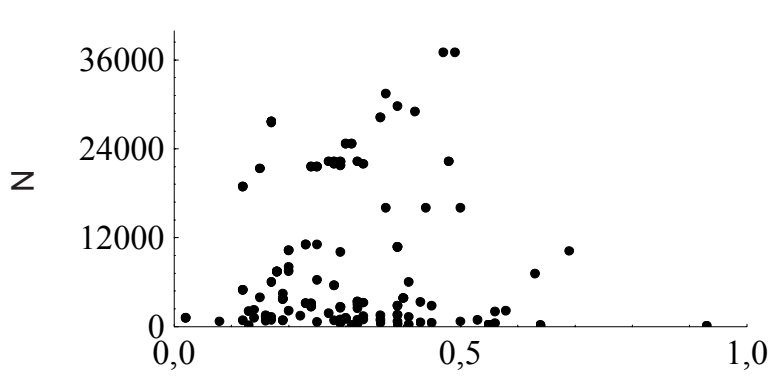

Herdabilidades P365

Heritabilities W365

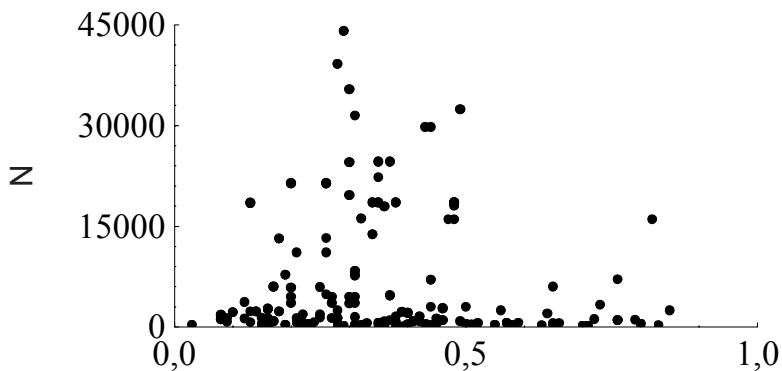

Herdabilidades P550 Heritabilities W550

Figura 2 - Gráficos de funil das estimativas de herdabilidade para as características: (a) peso ao nascimento (PN); (b) peso à desmama (PD); (c) peso aos 365 dias (P365); (d) peso aos 550 dias (P550).

Figure 2 - Funil plots of the heritability estimates for the traits: (a) birth weight (BW); (b) weaning weight (WW); (c) weight at 365 days (W365); (d) and weight at 550 days (W550).

Tabela 1 - Estatística $Q$ com nível de significância e grau de liberdade para as características de crescimento

Table 1 - $Q$ statistic with significant level and degree of freedom for the growth traits

\begin{tabular}{lcc}
\hline $\begin{array}{l}\text { Característica } \\
\text { Trait }\end{array}$ & $\begin{array}{c}\text { Grau de liberdade } \\
\text { Degree of freedom }\end{array}$ & $Q$ \\
\hline $\begin{array}{l}\text { Peso ao nascimento } \\
\text { Birth weight }\end{array}$ & 181 & $619,65^{* *}$ \\
$\begin{array}{l}\text { Peso à desmama } \\
\text { Weaning weight }\end{array}$ & 330 & $1205,20^{* *}$ \\
$\begin{array}{l}\text { Peso aos 365 dias } \\
\text { Weight at 365 days }\end{array}$ & 152 & $669,12^{* *}$ \\
$\begin{array}{l}\text { Peso aos 550 dias } \\
\text { Weight at 550 days }\end{array}$ & 202 & $744,01 * *$ \\
\hline
\end{tabular}

${ }^{* *}$ Significativo pelo teste $Q(p \leq 0,01)$ (Significant by $Q$ test, $p \leq 0.01$ ).
Tabela 2 - Estimativas de herdabilidades combinadas $\left(\hat{h}_{+}^{2}\right)$, com respectivo desvio-padrão $\left(\hat{s}_{+}\right)$, para as características peso ao nascimento (PN), peso à desmama (PD), peso aos 365 dias (P365) e peso aos 550 dias (P550)

Table 2 - Pooled heritability estimates $\left(\hat{h}_{+}^{2}\right)$, with standard deviation $\left(\hat{S}_{+}\right)$, for birth weight $(B W)$, weaning weight (WW), weight at 365 days old (W365) and weight at 550 days old (W550)

Estimativa combinada Característica

Pooled estimate

Trait

\begin{tabular}{lcccc} 
& PN & PD & P365 & P550 \\
& $B W$ & $W W$ & $W 365$ & $W 550$ \\
\hline$\hat{h}_{+}^{2}$ & 0,30 & 0,23 & 0,27 & 0,31 \\
$\hat{s}_{+}$ & 0,01 & 0,01 & 0,01 & 0,01 \\
\hline
\end{tabular}


cruzamentos, obtiveram $0,34 \pm 0,00$ para $\mathrm{PN}$, de $0,30 \pm 0,00$ para $\mathrm{PD}, 0,37 \pm 0,01$ para $\mathrm{P} 365$ e $0,31 \pm 0,02$ para P550. Verifica-se, a partir destes valores que, exceto para o valor de referência da estimativa de herdabilidade para P550, que foi superior ao obtido neste trabalho, tanto os valores de referência de herdabilidade quanto as $\hat{h}_{+}^{2}$ obtidas por esses autores, apresentaram valores bastante próximos aos encontrados neste trabalho. Ressalta-se, ainda, que os desvios-padrão mais baixos encontrados pelos auto-

Tabela 3 - Estimativas combinadas das herdabilidades e número de estimativas, entre parênteses, das características peso ao nascimento (PN), peso à desmama (PD), peso aos 365 dias (P365) e peso aos 550 dias (P550) por raça, país e método de estimação ( $M A=$ =modelo animal; TOURO=modelo touro; RPP=regressão progênie-pai)

Table 3 - Pooled heritability estimates and number of estimates between parenthesis of birth weight (BW), weaning weight (WW), weight at 365 days of age (W365) and weight at 550 days of age (W550) by breed, country, and estimation method (AM=animal model; SIRE=sire model; OPR=offspring-parent regression)

\begin{tabular}{|c|c|c|c|c|}
\hline \multirow[t]{2}{*}{$\begin{array}{l}\text { Raça } \\
\text { Breed }\end{array}$} & \multicolumn{4}{|c|}{$\begin{array}{c}\text { Característica } \\
\text { Trait }\end{array}$} \\
\hline & $\mathrm{PN}(B W)$ & $\mathrm{PD}(W W)$ & P365 (W365) & P550 (W550) \\
\hline Afrikaner & $0,52(2)$ & $0,23(2)$ & $0,17(2)$ & $0,17(2)$ \\
\hline Bali & $0,22(1)$ & $(0)$ & $(0)$ & $(0)$ \\
\hline Boran & $0,17(6)$ & $0,22(2)$ & $(0)$ & (0) \\
\hline Brahman & $0,26(20)$ & $0,21(53)$ & $0,28(8)$ & $0,29(35)$ \\
\hline Bunaji & $(0)$ & $0,34(2)$ & $(0)$ & $(0)$ \\
\hline Gir & $0,36(8)$ & $0,19(10)$ & $0,21(2)$ & $0,17(3)$ \\
\hline Gobra & $0,14(2)$ & $0,26(2)$ & $0,35(2)$ & $0,18(4)$ \\
\hline Gudali & $0,33(3)$ & $0,15(3)$ & $0,37(1)$ & $0,31(1)$ \\
\hline Guzerá & $0,17(22)$ & $0,21(33)$ & $0,21(19)$ & $0,19(18)$ \\
\hline Indu & $0,24(1)$ & $0,07(1)$ & $(0)$ & $(0)$ \\
\hline Kedah & $0,18(1)$ & (0) & $(0)$ & (0) \\
\hline Mashona & $(0)$ & $0,28(3)$ & $(0)$ & $(0)$ \\
\hline Nelore & $0,32(105)$ & $0,24(201)$ & $0,27(107)$ & $0,32(129)$ \\
\hline Sahiwal & $0,06(1)$ & $(0)$ & $0,41(1)$ & $0,65(1)$ \\
\hline Tabapuã & $0,33(5)$ & $0,23(11)$ & $0,23(7)$ & $0,19(6)$ \\
\hline Wakwa & $0,62(2)$ & $0,29(2)$ & (0) & $(0)$ \\
\hline Zebu & $0,53(3)$ & $0,24(6)$ & $0,40(4)$ & $0,54(4)$ \\
\hline \multicolumn{5}{|l|}{ País } \\
\hline \multicolumn{5}{|l|}{ Country } \\
\hline África do Sul & $0,52(2)$ & $0,23(2)$ & $0,17(2)$ & $0,17(2)$ \\
\hline Austrália & (0) & $0,43(9)$ & $0,30(3)$ & $0,35(24)$ \\
\hline Brasil & $0,31(138)$ & $0,24(254)$ & $0,27(138)$ & $0,31(156)$ \\
\hline Camarões & $0,47(4)$ & $0,28(4)$ & (0) & (0) \\
\hline Colômbia & $0,24(2)$ & $0,18(5)$ & $(0)$ & $0,15(1)$ \\
\hline Costa Rica & (0) & $0,27(1)$ & (0) & (0) \\
\hline Cuba & $0,21(4)$ & $0,11(14)$ & $0,19(2)$ & $0,31(1)$ \\
\hline EUA & $0,36(3)$ & $0,25(11)$ & $0,13(1)$ & $0,22(1)$ \\
\hline Etiópia & $0,11(2)$ & $0,22(2)$ & $(0)$ & $(0)$ \\
\hline Guatemala & $(0)$ & $0,26(2)$ & $(0)$ & $(0)$ \\
\hline Guiana & $0,35(1)$ & $0,57(2)$ & $0,93(1)$ & $(0)$ \\
\hline Malásia & $0,20(2)$ & $(0)$ & $(0)$ & $(0)$ \\
\hline México & $0,27(3)$ & $0,37(4)$ & $0,30(1)$ & $0,72(1)$ \\
\hline Nigéria & $0,28(1)$ & $0,31(1)$ & $0,37(1)$ & $0,31(1)$ \\
\hline Paquistão & $0,06(1)$ & $(0)$ & $0,41(1)$ & $0,65(1)$ \\
\hline Senegal & $0,14(2)$ & $0,32(2)$ & $0,35(2)$ & $0,18(4)$ \\
\hline Tanzânia & $0,39(4)$ & $(0)$ & (0) & (0) \\
\hline Venezuela & $0,25(11)$ & $0,11(12)$ & $0,50(1)$ & $0,18(9)$ \\
\hline Zimbábue & $0,28(2)$ & $0,27(6)$ & (0) & $0,24(2)$ \\
\hline \multirow{2}{*}{\multicolumn{5}{|c|}{$\begin{array}{l}\text { Método } \\
\text { Method }\end{array}$}} \\
\hline & & & & \\
\hline $\mathrm{MA}(A M)$ & $0,31(89)$ & $0,24(196)$ & $0,27(102)$ & $0,31(135)$ \\
\hline TOURO (SIRE) & $0,29(83)$ & $0,21(123)$ & $0,26(51)$ & $0,29(62)$ \\
\hline $\mathrm{RPP}(P O R)$ & $0,22(10)$ & $0,19(12)$ & & $0,21(6)$ \\
\hline
\end{tabular}


res na obtenção das $\hat{h}_{+}^{2}$ podem ser decorrentes da utilização da ponderação $w_{i}$, a qual não adiciona a variância entre os estudos à variância individual das estimativas de herdabilidade.

$\mathrm{Na}$ análise de mínimos quadrados, os fatores que afetaram de maneira estatisticamente significativa $(\mathrm{p} \leq 0,05)$ as $\hat{h}_{+}^{2}$ ponderadas por $w_{i}^{*}$ foram raça e método de estimação, nas quatro características, e país, nas características PD e P550.

Koots et al. (1994a), utilizando um modelo com os fatores raça, país, origem dos dados (dados de experimentos ou de campo), manejo alimentar (pasto ou confinamento), método de estimação, sexo e data de publicação dos resultados, verificaram efeito estatisticamente significativo $(\mathrm{p} \leq 0,01)$ dos fatores: raça e sexo, para as $\hat{h}^{2}$ ponderadas da característica $\mathrm{PN}$; raça, método e sexo para as $\hat{h}^{2}$ ponderadas da característica PD; e manejo alimentar, para as $\hat{h}^{2}$ ponderadas da característica P365. Entretanto, os autores esperavam que o método de análise fosse significativo para a maioria das características analisadas. Nas análises de modelo animal, quando a matriz de relações aditivas é completa e vai até a população-base, as estimativas de variância não são afetadas pela seleção e são, portanto, maiores que aquelas estimadas por outros métodos. Por sua vez, as causas do efeito de país não estão claras e alguns países podem ter $\hat{h}^{2}$ mais altas, em virtude de diferentes práticas de manejo. Koots et al. (1994a) ressaltam ainda um provável confundimento entre raça e país, fato também notado neste trabalho.

Lôbo et al. (2000), empregando um modelo com os fatores método de estimação e grupo genético, encontraram significância estatística $(p \leq 0,05)$ somente para os fatores grupo genético nas características $\mathrm{PN}$ e P365, e método de estimação na característica PD.

As estimativas combinadas das herdabilidades calculadas utilizando a ponderação $w_{i}^{*}$ e divididas por raça, país e método de estimação, para as quatro características em estudo (PN, PD, P365 e P550), encontram-se na Tabela 3. Para os fatores raça e país, apesar de terem sido obtidas $\hat{h}_{+}^{2}$ em muitas situações, estas não representam uma média ponderada e sim uma estimativa pontual, uma vez que se tem apenas uma estimativa de herdabilidade para muitas raças e países, dificultando uma conclusão mais geral para estes fatores. Para o fator método, as $\hat{h}_{+}^{2}$ obtidas com modelo animal, como esperado, foram mais altas que as estimadas pelos outros dois métodos.

\section{Conclusões}

A meta-análise é um procedimento recomendável para obtenção de valores médios de estimativas de herdabilidade em características de crescimento em bovinos de corte de origem indiana, porém, é indispensável a apropriada análise do conjunto de dados a serem resumidos, pois as pressuposições requeridas validarão os resultados finais.

A variabilidade entre as estimativas de herdabilidade é importante e influencia na obtenção dos valores combinados de herdabilidade, devendo ser considerada na análise.

O método de estimação da herdabilidade e a raça dos animais foram os fatores significativos sobre as estimativas de herdabilidade ponderadas das quatro características.

\section{Agradecimento}

Ao Conselho Nacional de Desenvolvimento Científico e Tecnológico $(\mathrm{CNPq})$, pelo financiamento e apoio ao desenvolvimento deste estudo.

\section{Literatura Citada}

BOURDON, R.M. Understanding animal breeding. Upper Saddle River: Prendice-Hall, 2000. 538p.

BOX, G.E.P.; COX, D.R. An analysis of transformations. Journal of the Royal Statistical Society, v.26, p.211252, 1964.

BECKETT, S.D.; LEAN, I.J. Gonadotrophin-releasing hormone in postpartum dairy-cattle: a meta-analysis of effects on reproductive efficiency. Animal Reproduction Science, v.48, p.93-112, 1997.

BROCKWELL, S.E.; GORDON, I.R. A comparation of statistical methods for meta-analysis. Statistics in Medicine, v.20, p.825-840, 2001.

CANNER, P.L. An overview of six clinical trials of aspirin in coronary heart disease. Statistics in Medicine, v.6, p.255263, 1987.

COOPER, H.M. Integrating research: a guide for literature reviews. 2.ed. Newbury Park: Sage, 1989. 157p.

DerSIMONIAN, R.; LAIRD, N. Meta-analysis in clinical trials. Controlled Clinical Trials, v.7, p.177-188, 1986.

EGGER, M.; SMITH, G.D. Meta-analysis: bias in location and selection of studies. British Journal of Medicine, v.316, 61-66, 1998.

ENGELS, E.A.; SCHMID, C.H.; TERRIN, N. et al. Heterogeneity and statistical significance in meta-analysis: an empirical study of 125 meta-analysis. Statistics in Medicine, v.19, p.1707-1728, 2000.

FAGARD, R.H.; STAESSEN, J.A.; THIJS, L. Advantages and disadvantages of the meta-analysis approach. Journal of Hypertension, v.14, supp1.2, p.9-13, 1996. 
FALCONER, D.S.; MACKAY, T.F.C. Introduction to quantitative genetics. Edinburgh: Addison Wesley Longman, 1996. 464p.

GIANNOTTI, J.G.; PACKER, I.U.; MERCADANTE, M.E.Z. Meta-análise para estimativas de correlação genética entre pesos ao nascer e desmama de bovinos. Scientia Agricola, v.59, n.3, p.435-440, 2002.

GLASS, G.V. Primary, secundary, and meta-analysis of research. Educational Researcher, v.6, p.3-8, 1976.

GUREVITCH, J.; HEDGES, L.V. Meta-analysis: combining the results of independent experiments. In: SCHEINER, S.M.; GUREVITCH, J. (Eds.) Design and analysis of ecological experiments. New York: Chapman \& Hall, 1993. p.378-398.

HEDGES, L.V.; OLKIN, I. Statistical methods for metaanalysis. London: Academic Press, 1985. 369p.

KOOTS, K.R.; GIBSON, J.P.; SMITH, C. et al. Analyses of published genetic parameter estimates for beef production traits. 1. Heritability. Animal Breeding Abstracts, v.62, n.5, p.309-338, 1994a.

KOOTS, K.R.; GIBSON, J.P.; SMITH, C. et al. Analyses of published genetic parameter estimates for beef production traits. 2. Phenotypic and genetic correlations. Animal Breeding Abstracts, v.62, n.11, p.826-853, 1994 b.

LÔBO, R.N.B; MADALENA, F.E.; VIEIRA, A.R. Average estimates of genetic parameters for beef and dairy cattle in Tropical regions. Animal Breeding Abstracts, v.68, p.433-462, 2000.

MERCADANTE, M.E.Z.; LÔBO, R.B.; REYES, A. Parámetros genéticos para características de crecimiento en cebuínos de carne: una revisión. Archivo Latinoamericano Producción Animal, v.3, n.1, p.45-89, 1995.

MOHIUDDIN, G. Estimates of genetic and phenotypic parameters of some trais in beef cattle. Animal Breeding Astracts, v.61, n.8, p.495-522, 1993.

NORMAND, S.T. Tutorial in biostatistics meta-analysis: formulating, evaluating, combining, and reporting. Statistics in Medicine, v.18, p.321-359, 1999.
OLKIN, I.; SHAW, D.V. Meta-analysis and its applications in horticultural science. Hort Science, v.30, n.7, p.1343-1348, 1995.

PICKARD, L.M.; KITCHENHAM, B.A.; JONES, P.W. Combining empirical results in software engineering. Information and Software Technology, v.40, p.811$821,1998$.

ROUGHSEDGED, T.; THOMPSON, R.; VILLANUEVA, B. et al. Synthesis of direct and maternal genetic components of economically important traits from beef breed-cross evaluations. Journal of Animal Science, v.79, p.23072319, 2001.

STATISTICAL ANALYSES SYSTEM - SAS. SAS/STAT software: changes and enhancements through release 6.12 . Cary: 1997. 1162p.

STATISTICAL ANALYSES SYSTEM - SAS. SAS/STAT user's guide. version 6.4. Cary: 1990. 846p.

STATISTICAL ANALYSES SYSTEM - SAS. SAS macro programs: boxcox - power transformations by Box-Cox method. 2002. http://www.math.yorku.ca/SCS/sasmac/ boxglm.html. (04 ago. 2003)

SHAPIRO, S.S.; WILK, M.B. An analysis of variance test for normality (complete samples). Biometrika, v.52, p.591$611,1965$.

St-PIERRE, N.R. Integrating quantitative findings from multiple studies using mixed model methodology. Journal of Dairy Science, v.84, p.741-755, 2001.

WANG, M.C.; BUSHMAN, B.J. Integration results: through meta-analytic review using SAS software. Cary: SAS Institute, 1999. 400p. 